\title{
Twenty-five years of HIV management
}

\author{
A Jayasuriya C Robertson P S Allan
}

J R Soc Med 2007; 100:363-366

\section{INTRODUCTION}

In 1981 the first case of AIDS was reported. ${ }^{1}$ A quarter of a century on, the AIDS epidemic has claimed the lives of more than 25 million people. It has rapidly become one of the most highly publicized, and politicized, infectious epidemics. Over these 25 years, we have also made remarkable advances in diagnosis and therapy. What follows is an update on the state of play at the end of 2006.

\section{HIV EPIDEMIOLOGY}

The World Health Organization (WHO) estimates the total number of people living with HIV globally to be the highest ever, at 40.3 million. In 2005 alone, 4.9 million people were newly diagnosed and 3.1 million died. HIV predominantly affects younger adults, with over $70 \%$ of individuals being aged 15-39 years at diagnosis; in addition, 2.3 million children worldwide are now HIV-positive. ${ }^{2}$

Although the worst-hit region remains Sub-Saharan Africa, with two thirds of all HIV-positive people in the world, the steepest increase in incidence has been in Eastern Europe and Central Asia (25\% rise in 2005), where intravenous drug use and commercial sex are the predominant factors fuelling the epidemic. The WHO predicts that several other countries, such as Indonesia and Pakistan, may also be on the verge of an epidemic. ${ }^{2}$

In contrast, as a result of sustained and determined public health efforts, some countries - such as Uganda, Kenya, urban areas in Zimbabwe and a few Caribbean countries - are reporting declining HIV infection rates. Similar trends have been seen in sex workers in Thailand and Cambodia, and intravenous drug users in Brazil and Spain. ${ }^{2}$

It is estimated that there are 58,300 HIV-infected people in the UK, a third of whom are as yet undiagnosed. The highest risk group remains men who have sex with men, although in $200552 \%$ of new infections in the UK were acquired heterosexually. Seventy per cent of these new diagnoses were in people of Sub-Saharan African origin. HIV transmission amongst intravenous drug users in the UK is currently low ( $2 \%$ of all new diagnoses). ${ }^{3}$

Department of Genitourinary Medicine, Coventry, and Warwickshire Hospital, Stoney Stanton Road, Coventry CV1 4FH, UK

Correspondence to: Dr Ashini Jayasuriya

Email: ashini.jayasuriya@coventrypct.nhs.uk
Vertical spread has also declined with the advent of routine antenatal testing, although preventable HIV transmission from mother to child still occurs in those undiagnosed at delivery.

\section{AIDS CO-INFECTION}

In 2006 we celebrated the anniversary of a major milestone in the history of HIV: the advent of Highly Active Antiretroviral Therapy (HAART) a decade ago revolutionized HIV treatment and has resulted in a dramatic decline of AIDS diagnoses and deaths. AIDS diagnoses are now primarily made in those presenting late with HIV infection. ${ }^{3}$ In the era of HAART, the spectrum of AIDS-defining illnesses is also changing, with proportionately more AIDSdefining tumours, such as non-Hodgkins lymphoma, and fewer infections associated with very low CD4 counts, such as CMV retinitis and Mycobacterium avium intracellulare. ${ }^{4}$ There continues to be a surge of HIV/tuberculosis (TB) coinfections, with a 5\% rise in Mycobacterium tuberculosis infections observed in the UK in the last year alone. ${ }^{5}$

\section{TREATMENT}

The sustained decline in HIV-associated deaths and the rise in new diagnoses have resulted in a steep rise in the number of people requiring long-term therapy.

Access to antiretrovirals has greatly improved over the past two years. Previously only available in the West, countries such as Brazil, Cuba, Chile and Argentina now have over $80 \%$ coverage. However, regions such as Asia, Africa and Eastern Europe still have extremely poor coverage, with at best only one in ten Africans and one in seven Asians who require HAART having access to any treatment at all. ${ }^{2}$

The WHO/UNAIDS ' 3 by 5 ' campaign had the aim of getting three million people living with HIV/AIDS in lowand middle-income countries on antiretroviral treatment by the end of 2005. Although this target has not yet been reached, the campaign has been a catalyst for improving antiretroviral access and still hopes to achieve universal access by $2010 .^{6,7}$

The indications for initiating antiretroviral therapy include a CD4 count less than 200 cells $/ \mathrm{mm}^{3}$ (although treatment may be considered at CD4 counts of 200-350), a diagnosis of AIDS or a related tumour, or severe recurrent HIV-related illness irrespective of CD4 count. $^{8}$ 
There are currently 22 individually approved anti-HIV drugs in the UK and four fixed-dose combination pills. A standard HAART regimen consists of two nucleoside reverse transcriptase inhibitors (NRTIs) with a non nucleoside reverse transcriptase inhibitor (NNRTI) or boosted protease inhibitor (PI). The British HIV Association (BHIVA) recommended regimens can be viewed at www.bhiva.org. In practice, a regimen is selected on the basis of dosing schedule, baseline resistance, hepatitis B or C co-infection, cardiovascular disease risk, psychiatric history, lifestyle issues and side-effect profiles. Concurrent renal disease, bone marrow suppression and hepatic disease may also be influential. Virological success is defined by the suppression of HIV viral load to less than 50 copies per $\mathrm{ml}$. The main determinant of successful and durable virological and immunological responses to HAART remains robust adherence, and multidisciplinary support needs to be available to patients in order to achieve this.

\section{New antiretrovirals}

The first new antiretroviral to be licensed in 2006 was Tipranivir - a PI thought to be useful in patients with multiple mutations to other PIs. Like most PIs, its efficacy is augmented with ritonavir, thereby reducing the side-effects associated with higher doses. At present it is being used in treatment-experienced patients, depending on their viral resistance profile. The main side effects are lipid and liver function test abnormalities and GI side effects. ${ }^{8}$

Two newer agents are being used under an expanded access scheme for named patients. One is a new PITMC114 (Darunavir) - useful in treatment-experienced patients with certain PI mutations, and the second is TMC125, an NNRTI with activity in patients with other NNRTI mutations.

CCR5 inhibitors are an entirely new class of drugs that are eagerly awaited. They work by blocking the binding of HIV to the CD4 T-cell, thereby preventing viral invasion. In 2005 there were three potential candidate drugs undergoing trials. Severe hepatotoxicity seen with one and concerns about the potency of a second has hindered progress, but research is ongoing with the third. ${ }^{9}$

Two important areas for continuing research are maturation inhibitors, which interfere with production of the viral capsular protein, and integrase inhibitors, which target integrase, the enzyme that splices HIV genes into the cellular DNA.

\section{HLA testing in predicting side effects of HAART}

Abacavir, a NRTI, is associated with a significant risk $(4.9 \%)$ of a hypersensitivity reaction that manifests as fever, dyspnoea, rash, nausea, vomiting, diarrhoea and abdominal pain, joint pain and lymphadenopathy. It is potentially fatal on re-exposure. ${ }^{10}$ HLA B5701 has been found to be strongly associated with the development of this reaction, and last year HLA-typing prior to initiation of Abacavir was introduced into clinical practice in some HIV clinics. Other HLA polymorphisms may potentially be influential, as some patients without HLA B5701 do exhibit hypersensitivity. The manufacturers of Abacavir are currently investigating the utility of HLA testing as a risk assessment tool. There is further opportunity for genetic testing to predict toxicity, exemplified by the association of Nevirapine-induced hepatotoxicity and DR0101.

\section{Resistance}

Owing to the rapid rate of viral replication and the high mutation rate of the HIV genome, the development of viral resistance remains one of the main obstacles to successful therapy. In a study of previously drug-naïve UK patients who started HAART between 1996 and 2003, there was a $38 \%$ risk of failure and a $27 \%$ risk of resistance over six years of follow up. ${ }^{11}$ The refinement and availability of resistance testing has enabled clinicians to make therapeutic decisions based on the presence of recognized mutations determined by PCR. However, there remains uncertainty about the exact correlation of some mutations and clinical outcome, so that a phenotypic resistance assay is sometimes used. Minority species of resistant virus may also be missed with conventional resistance testing; hence caution needs to be exercised when interpreting results.

Perhaps more disconcertingly, primary resistance in the UK is quoted at $5-26 \% .{ }^{8}$ The presence of primary resistance compromises the speed and efficacy of response to first-line therapy, promotes the development of further mutations and compromises future treatment options. BHIVA now recommends that all patients due to start antiretrovirals undergo a baseline viral resistance assay.

\section{DEVELOPMENTS IN HIV PREVENTION}

\section{Post-exposure prophylaxis, including following sexual exposure}

There is thought to be a window of opportunity to abort HIV infection by promptly inhibiting viral replication following exposure. For this reason, post-exposure prophylaxis has been used widely following occupational exposure. Retrospective case controlled studies of AZT given to health care workers post-exposure ${ }^{12}$ and to neonates post-delivery ${ }^{13}$ have shown a protective effect. There are no human studies to prove the benefit of HAART as PEP, but as triple therapy is more effective than monotherapy in reducing viral load, logic dictates that it is likely to be the superior option. 
Guidelines for post exposure prophylaxis following sexual exposure (PEPSE) were published earlier this year by the British Association for Sexual Health and HIV (BASHH). The risk of transmission is dependent on several factors, including the risk that the source is positive, characteristics of the exposure, degree of infectivity of the source and host susceptibility. Every effort should be made to establish the status of the source. Aggravated intercourse (such as sexual assault), concurrent STIs and high HIV viral loads result in higher transmission risk. Receptive anal intercourse involves a higher transmission risk $(0.1-3.0 \%$ per exposure) than vaginal intercourse $(0.1-0.2 \%)$ and similarly vaginal intercourse involves a higher risk than oral intercourse (0-0.04\%). ${ }^{14}$

As antiretrovirals can have serious side effects and as yet unknown potential long-term complications, the risks and benefits of PEP(SE) need to be balanced for each case. However, treatment should be initiated as soon as possible and within 72 hours of exposure.

The adherence of patients on PEP(SE) is significantly poorer than in HIV-positive patients on treatment, which may compromise drug efficacy and theoretically cause drug resistance should the patient seroconvert. Patient motivation, support and counselling are therefore very important at during the initiation of therapy.

Starter packs, available in accident and emergency departments and genitourinary medicine clinics will usually contain two NRTIs and a PI. As available evidence is based on zidovudine, it is usually included. The actual make up of the treatment will vary depending on local guidelines, knowledge about drug resistance in the source and the clinical background of the patient. Screening for other sexually transmitted infections and hepatitis B prophylaxis should not be forgotten.

Once given PEP, seroconversion may be delayed for longer than the usual 12-week period, so HIV serology should be checked at six weeks, three months and six months post event.

\section{Male circumcision}

A South African study recently demonstrated a $60 \%$ reduction in the incidence of HIV transmission in men who had been circumcised compared with uncircumcised controls. Although further studies need to be done before male circumcision can be advocated as a prevention tool, this may potentially be a cheap and globally accessible preventive aid. ${ }^{2}$

\section{Vaccination}

In the long term, our best hope for bringing the HIV epidemic under control is the development of a vaccine. Currently there are 23 potential vaccines undergoing trials, with four agents in phase II trials and one in phase III trials. The development of a successful vaccine remains hindered by scientific challenges, inadequate resources and intellectual property issues.

\section{PREGNANCY}

17.5 million women in the world live with HIV - an increase of one million in the past two years. $77 \%$ of these women live in Sub-Saharan Africa, with profound implications on vertical transmission in these resourcelimited countries. In six southern African countriesLesotho, South Africa, Namibia, Swaziland, Zimbabwe and Botswana - the prevalence of HIV in pregnant women exceeds $20 \% .^{2}$

The prevalence of HIV infection in pregnancy in the UK continues to rise, reaching levels of 1 in 180 in some innerLondon areas. In 1999, the Department of Health recommended HIV testing in every pregnant woman, which led to a huge increase in women aware of their diagnosis and drastically reduced the rates of infants being vertically infected. Without intervention the rate of vertical transmission is $35 \% .{ }^{13}$ This is reduced to $<1 \%$ with HAART, caesarean section, AZT infusion at delivery, avoidance of breastfeeding, and PEP for the neonate for four weeks post-delivery. In women who are fully virologically suppressed at the time of delivery, there is data to support vaginal delivery with equivalent transmission rates to caesarean section. ${ }^{16}$

\section{LONG-TERM CONSIDERATIONS IN THE MANAGEMENT OF HIV}

With the advances in HIV therapy, the life expectancy of HIV-positive patients has significantly improved in the West. Now treated more like a chronic disease than a death sentence, physicians involved in HIV care have the opportunity to think about long-term effects when planning management.

An increasing prevalence of fat re-distribution, diabetes and dyslipidaemia is seen in HIV-positive patients on HAART, with the prevalence of metabolic syndrome being significantly higher in patients with HIV. The risk of cardiovascular disease is three times higher in patients with metabolic syndrome and observational studies have suggested an association between HIV, HAART and cardiovascular disease. ${ }^{17}$ Although the chronic inflammatory effects of HIV itself may account for at least some of the increased risk, exposure to HAART (particularly PIs) has directly been associated with an increase in cardiovascular events. ${ }^{18}$

It is also quite striking that approximately two-thirds of HIV-positive men aged 17-40 smoke cigarettes, compared to one-third in age-matched controls. It is therefore crucial to assess lifestyle and cardiovascular risk factors during the initial work up of a newly diagnosed HIV patient. 
As some newer PIs have better lipid profiles, these longterm implications are taken into consideration when devising a HAART regime. Similarly, antiretrovirals with a higher risk of lipodystrophy, such as stavudine or certain PIs, can be avoided or substituted in order to avoid the psychological and social morbidity that may be involved in the development of body shape changes with long-term HIV and antiretroviral therapy.

\section{CONCLUSIONS}

HIV medicine has developed at a phenomenal rate over the quarter century since the first AIDS patient was diagnosed in 1981. As clinicians sub-specialize in areas of particular concern, for example metabolic disorders or viral resistance, the speciality is expanding further and improving both the short- and long-term prognosis and care of HIVpositive patients. It is hoped that, with time, the stigma and fears surrounding HIV will fade and improved accessibility to both testing and treatment will impact favourably upon everyone in the world affected by the epidemic. In the meantime, every effort should be made to increase awareness about HIV prevention and treatment on a global scale as, while we wait for a vaccine, the number of HIVpositive patients escalates each day. We simply cannot afford to wait another 25 years for the tide to turn.

Competing interests $\mathrm{AJ}$ and $\mathrm{CR}$ have no interests to declare. PSA has received sponsorship from various pharmaceutical companies to attend educational conferences.

\section{REFERENCES}

1 Centers for Disease Control. Pneumocystis Pneumonia-Los Angeles. Morb Mort Weekly Rep 1981; 30: 1-3

2 WHO/UNAIDS. AIDS Epidemic Update 2005. WHO/UNAIDS, 2005. Available at www.who.int/hiv/epiupdates (accessed April 2006)

3 The Health Protection Agency. Epidemiological data/Surveillance Methods. The Health Protection Agency. Available at www.hpa.org.uk/ infections/topics_az/ hiv_and_sti/hiv/hiv.htm (accessed April 2006)
4 Mocroft A, et al. AIDS across Europe, 1994-98: the EuroSIDA Study. Lancet 2000;356:291-6

5 The Health Protection Agency. TB Update March 2006, TB Surveillance Update 2000-2004. The Health Protection Agency. Available at www.hpa.org.uk/infections/topics_az/tb/pdf/newsletter_2006.pdf (accessed April 2006)

6 WHO. Treating 3 million by 2005. Making it happen. The WHO strategy, 2003. Available at www.who.int/3by5/publications/ documents (accessed April 2006)

7 WHO. 3 by 5 progress reports. WHO/UNAIDS. June 2005. Available at www.who.int/3by5/en (accessed April 2006)

8 British HIV Association guidelines for the treatment of HIV-infected adults with anti-retroviral therapy (2005). HIV Med 2005;6(Suppl 2): $1-61$

9 Bernard E. Do CCR5 inhibitors have a future? Aids Treatment Update $2006 ; 153$

10 Berenguer, et al. Effectiveness and safety of abacavir, lamivudine, and zidovudine in antiretroviral therapy-naive HIV-infected patients: results from a large multicenter observational cohort. J AIDS 2006; 41:154-9

11 UK collaborative Group on HIV Drug Resistance, UK CHIC Study Group. Long term probability of detection of HIV-1 drug resistance after starting antiretrovirals in routine clinical practice. AIDS 2005;19: 487-94

12 Cardo DM, et al. A case controlled study of HIV seroconversion in health care workers after percutaneous exposure to HIV infected blood: clinical and public health implications. N Engl J Med 1997;337: 1485

13 Sperling RS, et al. Maternal viral load, zidovudine treatment and the risk of transmission of Human Immunodeficiency virus type 1 from mother to infant. $N$ Engl J Med 1996;335:1621-9

14 Fisher M, et al. BASHH guideline: UK guideline for the use of postexposure prophylaxis for HIV following sexual exposure, Int J STD AIDS 2006;17:81-92

15 British HIV Association. Guidelines for the Management of HIV Infection in Pregnant Women and the Prevention of Mother-to-Child Transmission of HIV. BHIVA, 31 March 2005. Available at www.bhiva.org (accessed in April 2006)

16 Connor E, et al. Reduction of maternal-infant transmission of HIV Type-1 with zidovudine therapy. N Engl J Med 1994;331:1173-80

17 Das S. HIV and increased risk of cardiovascular diseases. Sexual Health 2005;2:219-21

18 Mary-Krause M, et al. Clinical Epidemiology Group from the French Hospital Database: Increased risk of myocardial infarction with duration of protease inhibitor therapy in HIV-infected men. AIDS 2003; 17:2479-86 